\title{
Quantifying nonadditive selection caused by indirect ecological effects
}

\author{
Casey P. terHorst, ${ }^{1,2,5}$ Jennifer A. Lau, ${ }^{1,3}$ Idelle A. Cooper, ${ }^{1,4}$ Kane R. Keller, ${ }^{1,3}$ Raffica J. La Rosa, ${ }^{1,3}$ \\ Anne M. Royer, ${ }^{1,3}$ Elizabeth H. Schultheis, ${ }^{1,3}$ Tomomi Suwa, ${ }^{1,3}$ and Jeffrey K. Conner ${ }^{1,3}$ \\ ${ }^{1}$ Kellogg Biological Station, Michigan State University, 3700 East Gull Lake Drive, Hickory Corners, Michigan 49060 USA \\ ${ }^{2}$ Department of Biology, California State University Northridge, 18111 Nordhoff Street, Northridge, California 91330 USA \\ ${ }^{3}$ Department of Plant Biology, Michigan State University, 612 Wilson Road, East Lansing, Michigan 48824 USA \\ ${ }^{4}$ Department of Biology, James Madison University, MSC 7801, Harrisonburg, Virginia USA
}

\begin{abstract}
In natural biological communities, species interact with many other species. Multiple species interactions can lead to indirect ecological effects that have important fitness consequences and can cause nonadditive patterns of natural selection. Given that indirect ecological effects are common in nature, nonadditive selection may also be quite common. As a result, quantifying nonadditive selection resulting from indirect ecological effects may be critical for understanding adaptation in natural communities composed of many interacting species. We describe how to quantify the relative strength of nonadditive selection resulting from indirect ecological effects compared to the strength of pairwise selection. We develop a clear method for testing for nonadditive selection caused by indirect ecological effects and consider how it might affect adaptation in multispecies communities. We use two case studies to illustrate how our method can be applied to empirical data sets. Our results suggest that nonadditive selection caused by indirect ecological effects may be common in nature. Our hope is that trait-based approaches, combined with multifactorial experiments, will result in more estimates of nonadditive selection that reveal the relative importance of indirect ecological effects for evolution in a community context.
\end{abstract}

Key words: Acmispon wrangelianus; adaptation; community context; competition; diffuse coevolution; indirect effects; Medicago polymorpha; natural selection; nonadditive selection; pairwise selection; plantherbivore interactions; plant-pollinator interactions.

\section{INTRODUCTION}

Species in natural communities interact with a multitude of other species, and these species interactions have important fitness consequences that affect both ecological and evolutionary dynamics. Although the effects of direct pairwise interactions with predators, competitors, and mutualists have been well explored, indirect effects among species can also be important in multispecies communities. Indirect ecological effects occur when a direct interaction between two species is altered by a third species (Strauss 1991, Wootton 1994). Indirect effects may be especially common in diverse communities, as the number of possible indirect effects increases exponentially with the number of species in a community (Abrams 1992), and can have important fitness consequences (Walsh 2013). In many cases, the magnitude of indirect ecological effects is greater than that of direct effects on species abundances, species coexistence, and community diversity (Vandermeer 1969, Stone and Roberts 1991, Lawler 1993, Miller 1994, Menge 1995). Although the ecological implica-

Manuscript received 31 March 2014; revised 6 February 2015; accepted 13 February 2015; final version received 5 March 2015. Corresponding Editor: B. D. Inouye.

${ }^{5}$ E-mail: casey.terhorst@csun.edu tions of indirect effects have been well studied, the evolutionary consequences have only recently gained attention (Walsh 2013).

One evolutionary consequence of indirect ecological effects is nonadditive selection (Strauss et al. 2005, Haloin and Strauss 2008). Nonadditive selection due to indirect ecological effects occurs when an indirect ecological effect alters the strength or direction of selection that one species imposes on another. In this case, selection on a given trait in the presence of two interacting species cannot be predicted from the additive effects of selection by each species alone. The frequency and importance of indirect ecological effects in nature suggests that nonadditive selection may be common. If so, selection in multispecies communities cannot be predicted from estimates of natural selection in pairwise interactions, and the magnitude or direction of selection on traits will vary in unpredictable ways across communities that differ in species composition. In pairwise interactions (direct ecological effects only), selection always results in adaptation to the selective agent, assuming that there is additive genetic variance for the trait under selection (Conner and Hartl 2004); however, in multispecies scenarios, nonadditive selection may either constrain or augment the rate of adaptation 


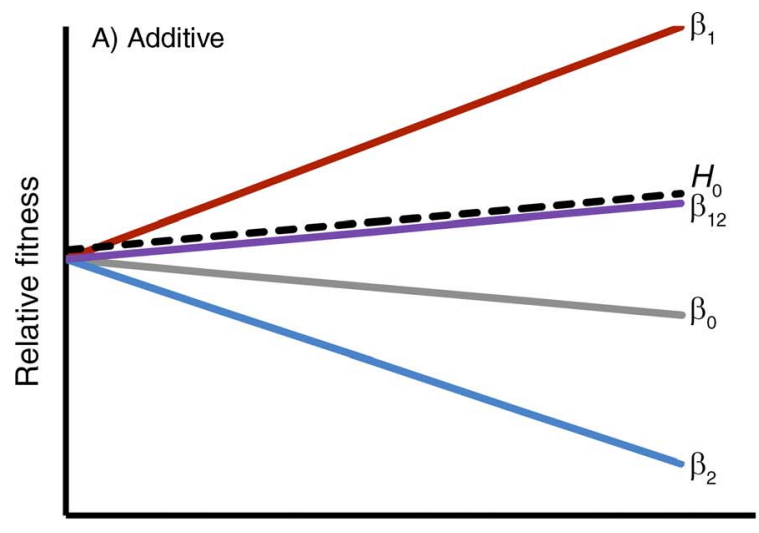

Trait value

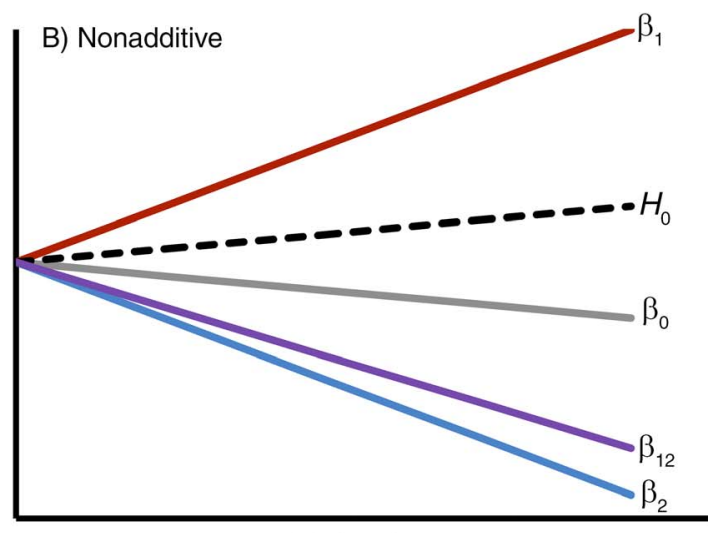

Trait value

FIG. 1. Examples of selection gradients when selection on a trait is (A) additive and (B) nonadditive. In these hypotheticals, there is weak selection against the trait when neither interactor is present $\left(\beta_{0}\right)$. Species 1 imposes positive selection on the trait $\left(\beta_{1}>\right.$ $\left.\beta_{0}\right)$. Species 2 imposes negative selection on the trait $\left(\beta_{2}<\beta_{0}\right)$. In $(A)$, selection is additive because neither herbivore alters the selection imposed by the other, and selection in the presence of both species $\left(\beta_{12}\right)$ is equivalent to the sum of their individual selective effects $\left(H_{0}=\beta_{1}+\beta_{2}-\beta_{0}\right)$. In (B), species 2 alters the selection imposed by species 1 , resulting in nonadditive selection because selection in the presence of both species differs from the sum of their individual effects $\left(\beta_{12}<H_{0}\right)$.

to any one particular interacting species compared to the pairwise scenario (Strauss et al. 2005).

As a hypothetical example, consider a plant population that primarily interacts with two herbivore species that impose selection on constitutive levels of a chemical defense (Fig. 1). Selection in natural communities can be estimated as the slope $(\beta)$ of the partial regression of relative fitness on standardized trait values (the standardized selection gradient; Lande and Arnold 1983; Fig. 1). In this hypothetical scenario, imagine there is weak selection for decreased chemical concentration in the absence of herbivores, due to a small cost of producing the chemical $\left(\beta_{0}\right)$. Herbivore 1 is a generalist that selects for increased chemical concentration $\left(\beta_{1}\right)$ because plants with higher chemical concentration experience less herbivory and consequently have higher relative fitness. Herbivore 2 is a specialist that is resistant to the chemical and uses it as a cue to locate plants; therefore, the specialist causes more damage on plants with high chemical concentration and selects for lower chemical concentration $\left(\beta_{2}\right)$. In a scenario where both herbivores are present and there are no indirect ecological effects, selection is additive (Fig. 1A); the selection gradients by the two herbivores are summed, resulting in very weak selection when both herbivores are present $\left(\beta_{12}\right)$. Selection is additive because neither herbivore alters the selection imposed by the other, and selection in the presence of both species can be predicted from pairwise selection by each herbivore. In this additive case, selection on the same trait in opposite directions results in a plant population that slowly adapts to Herbivore 1 and becomes maladapted to Herbivore 2.

In this hypothetical example, indirect ecological effects would result if the presence of one herbivore alters interactions between the plant and the other herbivore, through many possible mechanisms. Consider the scenario where, in the presence of specialist herbivores, generalist herbivores are deterred from feeding. In our example, the presence of specialist Herbivore 2 now reduces the selective effect imposed by generalist Herbivore 1 on chemical defense. The result is that selection in the presence of both herbivores $\left(\beta_{12}\right)$ is similar to selection in the presence of only Herbivore 2 ( $\beta_{2}$; Fig. 1B). Selection on chemical defense is nonadditive in this case, because selection in the presence of both species is not equivalent to the sum of their individual pairwise selection gradients. In this multispecies scenario, selection on chemical concentration results in adaptation to Herbivore 2, but results in maladaptation to Herbivore 1 (Fig. 1B), the opposite pattern of the additive case (Fig. 1A). Nonadditive selection will always affect adaptation to one or more species, but the exact way in which this occurs depends on the ecological scenario (see Appendix C: Fig. C1 for additional scenarios).

Few, if any, studies have estimated the strength of, or even directly tested for, nonadditive selection (but see Sahli and Conner 2011). Thus, we cannot yet conduct a review or meta-analysis of such studies. However, we believe that with existing data or future experiments, one could easily test for nonadditive selection with minimal additional effort. We suggest a statistical framework for quantifying the strength of nonadditive selection due to indirect ecological effects relative to pairwise selection in which selection occurs only in response to the direct effects of a single interactor. This method can be used for comparisons within and across studies and for identifying how and when indirect ecological effects are likely to influence evolution. We identify a small number of studies that performed the necessary experimental manipulations and analyses to formally test for nonad- 
ditive selection, although none interpreted their findings in the context of nonadditive selection (Juenger and Bergelson 1998, Stinchcombe and Rausher 2001, 2002, Lankau and Strauss 2008, Lau 2008, but see Sahli and Conner 2011). As we describe further below, an appropriate design requires multifactor manipulative experiments, which many ecologists already commonly employ to test for indirect ecological effects, among other reasons. Additionally, trait-based approaches to understanding communities (e.g., Ackerly and Cornwell 2007, Litchman and Klausmeier 2008) and calls to examine intraspecific trait variation in ecological studies (Violle et al. 2012) have garnered attention in recent years. These studies often report only mean traits of populations rather than individual fitness and trait values, although the latter data may be available in some cases. Our hope is that trait-based approaches, combined with multifactorial experiments, will result in more estimates of nonadditive selection that reveal the relative importance of indirect ecological effects for evolution in a community context.

\section{EXPERIMENTAL Design}

\section{Manipulating multiple species}

A multitude of studies have estimated selection gradients, but most are conducted in a single environment and cannot conclusively identify the selective agent (MacColl 2011). Experimentally manipulating the presence of species is the only way to definitively determine whether a species is a selective agent and to estimate the strength of selection imposed by that species (Wade and Kalisz 1990). Therefore, the ideal test for nonadditive selection requires experimental manipulations of multiple species to identify selective agents and determine how selection differs in a multispecies context vs. each pairwise context. The strength of selection in each manipulated environment can be estimated using selection gradients (the slope of the partial regression of relative fitness on standardized trait values; Lande and Arnold 1983) or variance standardized selection differentials (the slope of the regression of relative fitness on variance standardized trait values in a univariate analysis, also called selection intensity, or equivalently, the covariance between relative fitness and a variance standardized trait; Conner and Hartl 2004). In this paper, we use $\beta$ to symbolize either selection differentials or gradients because our approach can apply to either. Our approach also applies to traits standardized by the mean rather than the variance, which has some advantages (Hereford et al. 2004), but in this case the univariate slope does not equal the standardized selection differential. Trait standardization enables comparison of the strength of selection across traits within a study and across studies (e.g., Kingsolver et al. 2001).

If a manipulated species is a selective agent, then the selection gradient in the presence of that species $\left(\beta_{i}\right)$ will differ from the selection gradient in the absence of that species $\left(\beta_{0}\right)$. When multiple antagonistic species have been manipulated, $\beta_{0}$ should be defined as the case when none of the manipulated species are present. Estimating $\beta_{0}$ can be difficult in cases where the trait of interest is a measure of species interaction strength, such as herbivore tolerance or resistance, or competitive ability, because for such traits, it is impossible to measure trait values in the absence of the interacting species. Genotypic selection analyses (sensu Rausher 1992) where trait values are assigned based on measurements made in other treatments can help circumvent this issue (see Experimental design: Case study 1). Defining $\beta_{0}$ may also be challenging in many cases of obligate mutualisms, such as self-incompatible plant-pollinator interactions, because there will often be zero fitness in the absence of the mutualist species. In such cases, $\beta_{0}$ may be more appropriately estimated in a treatment in which the mutualism services are provided equally to all experimental organisms by the researcher. In pollination studies, for instance, flowers are sometimes handpollinated to the point of saturation to eliminate fitness differences caused by differential pollination (e.g., Galen 1996, Caruso et al. 2010). Regardless of how $\beta_{0}$ is defined, a factorial manipulation of multiple species that allows for estimation of $\beta_{0}$ is necessary, both to determine which species are selective agents and to accurately calculate a null hypothesis.

If there is natural variation among communities in the presence and absence of species, or if species composition can be experimentally manipulated, then the statistical approach we outline below can be used to quantify nonadditive selection. Because we are interested in indirect ecological interactions among species, we focus here on biotic agents of selection, but note that abiotic forces may also play a role in nonadditive selection, particularly when species interaction strengths vary across abiotic environments.

\section{Constructing a null hypothesis to test for nonadditive selection}

Testing for nonadditive selection requires a null hypothesis that reflects additive selection $\left(H_{0}\right.$ in Fig. 1). Two different methods have been proposed to generate the null expectation. Sahli and Conner (2011) estimated selection on floral traits in wild radish in response to honey bees, bumble bees, butterflies, and a combination of all three species. In pairwise treatments, each of the three pollinator species selected for increased anther exsertion. The additive null hypothesis of Sahli and Conner (2011) was based on the weighted average of the three pairwise selection gradients, as suggested by Strauss et al. (2005). Selection in the presence of multiple species was similar to the null hypothesis, leading to a conclusion of additive selection. In contrast, terHorst (2010) used a different null hypothesis when he measured the response to selection on protozoan cell size after dozens of generations of selection by either mosquito larvae predators, competition from other 
protozoa, or both predators and competitors. Protozoa evolved decreased cell size in response to both predators (terHorst et al. 2010) and competitors (terHorst 2011). In this case, the null hypothesis of additive selection was the sum of the pairwise effect sizes of predators and competitors on cell size, rather than the average effect size, and predicted the evolution of even smaller cell sizes when both competitors and predators were present. Cell size in the multispecies treatment was significantly different from the predicted additive effect (i.e., cell sizes were larger than predicted), indicating nonadditive response to selection resulting from strong indirect ecological effects (terHorst 2010).

We advocate the latter approach for constructing the null hypothesis; that is, the appropriate null hypothesis requires summing pairwise selection gradients. Selection is additive and can be predicted from the summed selection gradients estimated in pairwise interactions only when no interacting species alters the selection imposed by another. Simply put, if each species selects for an increased trait value, but those species have no effect on selection imposed by one another, then we expect selection on that trait to be stronger when both species are present. Consider the strength of selection imposed by each of two species, which are obtained by subtracting background selection due to unmanipulated factors:

$$
\begin{aligned}
& \beta_{\text {pairwise } 1}=\beta_{1}-\beta_{0} \\
& \beta_{\text {pairwise2 }}=\beta_{2}-\beta_{0} .
\end{aligned}
$$

These net pairwise selection gradients estimate the selection imposed due to direct ecological effects from each interacting species. If the effects of these species are additive, we propose that the predicted selection when both species are present is the sum of the net selection gradients of each species plus the background selection occurring when neither species is present:

$$
\begin{aligned}
H_{0} & =\beta_{\text {additive }}=\beta_{0}+\beta_{\text {pairwise } 1}+\beta_{\text {pairwise } 2} \\
& =\beta_{0}+\left(\beta_{1}-\beta_{0}\right)+\left(\beta_{2}-\beta_{0}\right)=\beta_{1}+\beta_{2}-\beta_{0} .
\end{aligned}
$$

Note the importance of $\beta_{0}$ in this calculation; if selection in the absence of both species $\left(\beta_{0}\right)$ is non-zero, then failure to account for such background selection will result in an incorrect estimate of the null hypothesis and potentially false conclusions about whether selection is additive or nonadditive. As more than two species are considered, $\beta_{0}$ becomes an even bigger component of Eq. 3, and failing to account for $\beta_{0}$ would result in an even greater deviation from the correct null hypothesis.

Previous work has relativized fitness either across all treatments, dividing by the grand mean fitness (e.g., Juenger and Bergelson 1998, Stinchcombe and Rausher 2001, 2002), or within each treatment, dividing by mean fitness within each treatment (e.g., Lankau and Strauss 2008, Sahli and Conner 2011). We evaluated both methods with a simple simulation model of species selecting on traits independently and additively (Appendix A). The simulation indicated that our proposed additive null model (Eq. 3) correctly predicts selection in the presence of both interactors when indirect ecological effects were absent and when fitness is relativized across all treatments. The model also reveals that nonadditive selection can arise via two mechanisms: (1) indirect ecological effects or (2) a change in mean fitness in the presence of multiple interactors that alters the opportunity for selection. The latter mechanism is not the result of an indirect ecological effect. Rather, for example, in the presence of multiple herbivores, fitness of all individuals may be reduced so that there is little variation in fitness among them and thus less opportunity for selection on plant traits, even if herbivores do not affect one another. Thus, such an effect can occur in the absence of indirect ecological effects.

When fitness is relativized within treatments, which causes all treatments to have equal mean relative fitness, patterns of nonadditive selection result from both indirect ecological effects and changes in mean fitness. In contrast, when fitness is relativized across all treatments, only indirect ecological effects produce nonadditive selection. A worthwhile goal for future work is a method for estimating the portion of nonadditive selection due to changes in mean fitness alone, similar to the estimates we produce here, or the portion due to indirect ecological effects alone, plus any portion due to the covariance between these two.

Ultimately, the decision of whether to relativize fitness within or among treatments depends on the question being asked and the mechanism of interest. If the goal is to make quantitative predictions of evolutionary responses and/or compare the strength of selection across studies, then one should use the approach of relativizing fitness within treatments and provide mean and variance estimates for traits and standard errors for selection gradients to facilitate future meta-analyses. Alternatively, if the goal is to predict the relative extent to which indirect ecological effects alter evolutionary responses, then one should relativize fitness across treatments. Fortunately, the two estimates can be converted with the equation (mathematical proof in Appendix B)

$$
\beta_{i \text { within }}=\left(\frac{\sigma_{z}}{\sigma_{i}}\right)\left(\frac{\bar{W}}{\bar{W}_{i}}\right) \beta_{i \text { across }}
$$

where $\beta_{i}$ represents the selection gradient in the presence of species $i$, with fitness relativized either within or among treatments, $\sigma_{\mathrm{z}}$ is the variance in traits across all treatments, $\sigma_{i}$ is the variance in traits within a treatment, $\bar{W}$ represents mean fitness across treatments, and $\bar{W}_{i}$ represents mean fitness within a treatment. If previous published studies provide means and variances of fitness and trait values, both within and among treatments, then those selection gradients $\left(\beta_{i \text { within }}\right)$ could also be

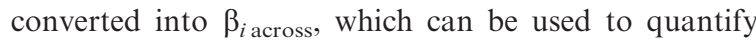
nonadditive selection using Eq. 5. As with all selection studies, we caution that comparing standardized selec- 
tion gradients across studies assumes that the individuals studied reflect the natural variation present in a population (Petraitis 1998).

\section{Estimating the relative strength of nonadditive selection}

Selection in a community context is not a dichotomy between additive and nonadditive. Rather, the degree to which selection is nonadditive is a continuum, as is the strength of selection caused by any pairwise interaction. Therefore, it is critical to quantify the strengths and uncertainties of nonadditive and pairwise selection. terHorst (2010) used the evolutionary responses of traits in pairwise treatments to calculate a predicted additive effect and estimated the strength of the nonadditive effect as the difference between the predicted additive effect and the evolutionary responses in the multispecies treatment. Using a similar approach for selection gradients, in a scenario where two species have been manipulated, we use

$$
\begin{aligned}
\beta_{\text {nonadditive }} & =\beta_{\text {multispecies }}-\beta_{\text {additive }}=\beta_{12}-\left(\beta_{1}+\beta_{2}-\beta_{0}\right) \\
& =\beta_{12}-\beta_{1}-\beta_{2}+\beta_{0} .
\end{aligned}
$$

This estimate provides both magnitude and direction of nonadditive selection. A positive nonadditive selection gradient indicates that selection in the presence of multiple interactors is more positive than expected from their pairwise effects. $\beta_{\text {nonadditive does not measure }}$ selection from any selective agent, but rather it quantifies how much selection is modified by indirect ecological effects. Eqs. 1 and 2 can be used to estimate pairwise selection, i.e., the direction and magnitude of selection imposed by direct effects of each species individually. In the next section, we describe how ANCOVA can be used to determine whether each of these estimates differ from zero; statistical programs, such as SAS, provide parameter estimates and standard

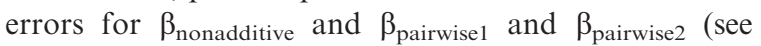
Supplement). These parameter estimates, along with uncertainties, are valuable because hypothesis testing is constrained by statistical power and thus subject to error, especially at smaller sample sizes.

\section{Using ANCOVA to test for nonadditive selection}

Analysis of covariance (ANCOVA) is ideal for testing the statistical significance of both pairwise selection by individual species and nonadditive selection. The factorial manipulation of multiple species that we recommend includes two fixed factors, the presence/absence of species 1 and the presence/absence of species 2, or even more factors when more species are manipulated. This full factorial design allows for estimates of $\beta_{0}$ and $\beta_{12}$, in addition to $\beta_{1}$ and $\beta_{2}$, so that one can determine whether each species is a selective agent and whether selection in the presence of multiple interactors is nonadditive. Alternatively, planned contrasts may be a viable option in designs that are not fully factorial (see Experimental design: Case study 2).
Three particular terms in the resulting ANCOVA output are important. The trait $\times$ species 1 interaction tests the overall effect of species 1 on selection on the trait; this interaction compares the average of $\beta_{0}$ and $\beta_{2}$ (i.e., species 1 absent) to the average of $\beta_{1}$ and $\beta_{1+2}$ (species 1 present). Similarly, the trait $\times$ species 2 interaction tests the overall effect of species 2 on selection on the trait by comparing the average of $\beta_{0}$ and $\beta_{1}$ to the average of $\beta_{2}$ and $\beta_{1+2}$. Finally, the threeway trait $\times$ species $1 \times$ species 2 interaction tests for nonadditivity (i.e., nonindependence) of the effects of species 1 and 2 on selection on the trait. A significant three-way interaction indicates that $\left(\beta_{1+2}-\beta_{2}\right) \neq\left(\beta_{1}-\right.$ $\beta_{0}$ ). By rearranging the terms, this inequality can also be expressed as $\beta_{1+2} \neq \beta_{1}+\beta_{2}-\beta_{0}$, which is the alternative hypothesis for testing for nonadditivity (cf. eq. 3).

We emphasize that these conclusions are subject to the assumptions of regression and ANCOVA (MitchellOlds and Shaw 1987). The most important assumptions are that errors are normally distributed and homoscedastic and that the means and variances of the continuous predictors (the traits in this case) do not differ greatly across the treatment groups. The latter should not be a problem if treatment groups are assigned randomly, except in cases where the traits are plastic in response to the treatment. Fitness estimates may often be nonnormally distributed or contain outliers, leading to heteroscedasticity. Although logtransformations can help in restoring normality, they also affect the biological interpretation of results (Lande and Arnold 1983, Stanton and Thiede 2005). In cases where assumptions are violated, resampling (e.g., bootstrapping) to generate $P$ values or generalized linear mixed models that incorporate non-Gaussian distributions are viable alternatives (Morrissey and Sakrejda. 2013).

Previous studies that manipulated the presence of multiple species in a full factorial design (Juenger and Bergelson 1998, Stinchcombe and Rausher 2001, 2002, Lankau and Strauss 2008) are appropriate for testing for nonadditive selection; two of these found significant trait $\times$ species $1 \times$ species 2 interactions indicative of nonadditive selection. Juenger and Bergelson (1998) quantified selection by multiple herbivores on flowering phenology in scarlet gilia (Ipomopsis aggregata) and found that the selective effects of seed flies and caterpillars were nonindependent. Selection on phenology was strongest in pairwise scenarios when either flies or caterpillars were present without the other; selection on phenology was weakest when both flies and caterpillars were present, resulting in a phenology $\times$ fly $\times$ caterpillar interaction. Here, $\beta_{\text {nonadditive }}$ would be negative. Antagonistic interactions between flies and caterpillars, or changes in plant quality when herbivores co-occur, may have created the indirect ecological effect that resulted in nonadditive selection (Juenger and Bergelson 1998). Such indirect ecological effects are responsible for the nonadditive selection in this study 
TABLE 1. ANCOVA results and planned contrasts from reanalysis of Lau (2008), examining the effects of Medicago, insects, resistance, and tolerance on seed production in Acmispon [formerly Lotus] wrangelianus.

\begin{tabular}{lccc}
\hline \hline \multicolumn{1}{c}{ Source } & Type III SS & $F_{1,283}$ & $P$ \\
\hline Resistance & & & \\
Medicago removal & 41.1 & 16.1 & $<\mathbf{0 . 0 0 1}$ \\
Insect reduction & 21.3 & 8.35 & $\mathbf{0 . 0 0 4 2}$ \\
Medicago $\times$ insects & 9.10 & 3.57 & 0.0598 \\
Resistance & 10.5 & 4.13 & $\mathbf{0 . 0 4 3 1}$ \\
Resistance $\times$ Medicago & 5.08 & 1.99 & 0.159 \\
Resistance $\times$ insects & 12.6 & 4.94 & $\mathbf{0 . 0 2 7 0}$ \\
Resistance $\times$ Medicago $\times$ insects & 10.7 & 4.20 & $\mathbf{0 . 0 4 1 4}$ \\
Tolerance & & & \\
Medicago & 32.2 & 11.7 & $<\mathbf{0 . 0 0 1}$ \\
Insects & 41.4 & 15.1 & $<\mathbf{0 . 0 0 1}$ \\
Medicago $\times$ insects & 2.22 & 0.810 & 0.370 \\
Tolerance & 9.60 & 3.49 & 0.0631 \\
Tolerance $\times$ Medicago & 0.0885 & 0.0300 & 0.858 \\
Tolerance $\times$ insects & 31.3 & 11.4 & $<0.001$ \\
Tolerance $\times$ Medicago $\times$ insects & 0.566 & 0.210 & 0.651 \\
\hline Notes: Fitness estimates were relativized across treatments. Boldface indicates significance of $P$ \\
$<$ 0.05. & & &
\end{tabular}

(rather than nonadditive selection resulting from treatment differences in mean fitness), because fitness was relativized across treatments.

Similarly, Lankau and Strauss (2008) measured selection by neighboring plants and molluscan herbivores on the concentration of a defensive chemical (sinigrin) in Brassica nigra. Selection by mollusks on sinigrin concentration was dependent on whether the neighbors of $B$. nigra were conspecifics or heterospecifics, resulting in a significant sinigrin $\times$ neighbor $\times$ mollusk interaction (Lankau and Strauss 2008). Because fitness was relativized within treatments in this study, this nonadditive selection could have been caused by indirect ecological effects, effects on mean fitness, or both. Stinchcombe and Rausher $(2001,2002)$ found that the presence of insects altered selection on deer resistance and tolerance in morning glory (Ipomoea hederacea), but that the effect of insects was similar regardless of whether fungi were present (nonsignificant tolerance $\times$ insects $\times$ fungi interaction). Thus, there were no indirect ecological interactions between insects and fungi that significantly altered selection on tolerance; in other words, selection from insects and fungi was largely additive. We now use two previously published case studies to illustrate how to calculate the strength of, and test for, nonadditive selection using the null model and methods we propose.

\section{Case study 1: nonadditive selection in response to herbivores and competitors}

Lau (2008) investigated how patterns of natural selection on herbivore tolerance and resistance in a native plant (Acmispon wrangelianus, formerly Lotus wrangelianus) were affected by insect herbivores and a competitor (the introduced plant Medicago polymorpha). This full factorial design, in which the abundance of insect herbivores and Medicago were experimentally manipulated in the field, is ideal for testing for nonadditive selection; we do so here by reanalyzing a subset of the data presented in Lau (2008).

The experiment included 2659 seedlings from 73 fullsib families that were sown into replicated Medicago removal $\times$ insecticide treatment plots $(N=44$ treatment plots; 11 plots per Medicago $\times$ insecticide treatment). Seed production and herbivory (proportion of leaflets damaged) were estimated for each individual. These data were used to estimate (1) a tolerance value for each family $[\ln$ (seed production in the presence of insects) $\ln$ (seed production in the absence of insects)], (2) a mean resistance value (1 - herbivory) for each family, and (3) a mean fitness value for each family in each Medicago removal $\times$ insecticide treatment. We applied ANCOVA to test whether selection on Acmispon defense traits (tolerance and resistance) by Medicago and insect herbivores was nonadditive due to indirect ecological effects (a significant trait $\times$ Medicago $\times$ insecticide interaction, with fitness relativized across treatments; SAS code in the Supplement).

ANCOVA indicated that selection on herbivore resistance was nonadditive (significant resistance $X$ Medicago $\times$ insecticide interaction; Table 1, Fig. 2A). There was selection against resistance in the absence of both Medicago and insect herbivores, presumably due to a cost of resistance $\left(\beta_{0}=-0.75 \pm 0.24\right.$; mean \pm SE; Fig. $2 \mathrm{~A})$. Selection was much weaker when either insects $\left(\beta_{1}\right.$ $=0.08 \pm 0.23)$ or Medicago $\left(\beta_{2}=-0.08 \pm 0.20\right)$ were present by themselves. We used Eqs. 1 and 2 to calculate the strength of selection imposed by each species; both Medicago and insects individually imposed selection for increased resistance $\left(\beta_{\text {insects }}=\beta_{1}-\beta_{0}=0.83 \pm 0.28\right.$; $\beta_{\text {Medicago }}=\beta_{2}-\beta_{0}=0.67 \pm 0.27$; mean $\pm \mathrm{SE}$ ). The selection for increased resistance imposed by both insects and competitors counteracted the negative selection in their absence, resulting in little overall 

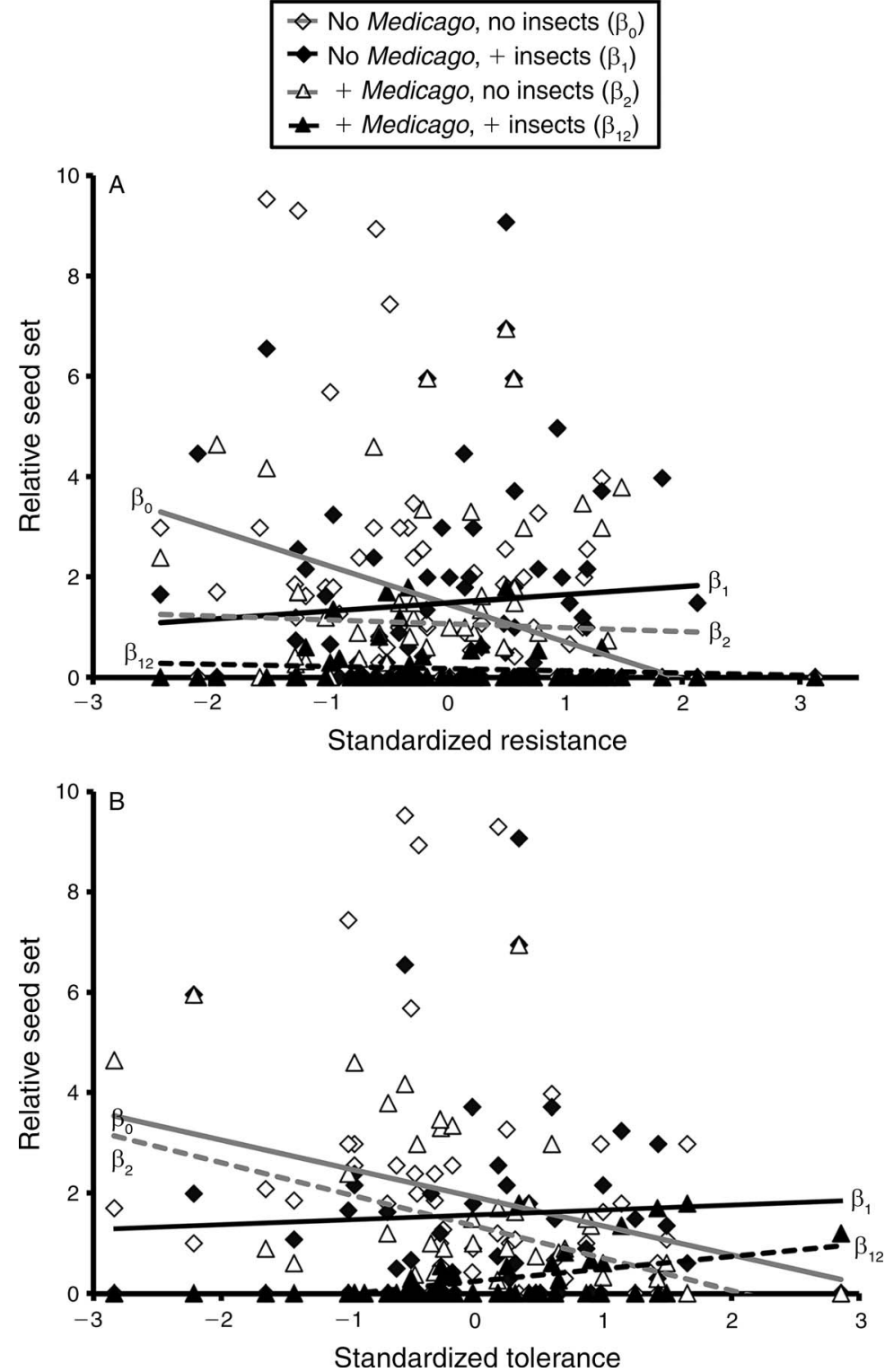

FIG. 2. Variance-standardized selection differentials, recalculated from Lau (2008), on Acmispon [formerly Lotus] wrangelianus (A) resistance and (B) tolerance. Selection on resistance is nonadditive, suggesting that selection imposed on resistance by insects is altered by the presence of Medicago. Selection on tolerance is additive, suggesting that insects, but not Medicago, are important selective agents on tolerance.

selection in the presence of each alone. If the effects of insects and Medicago were additive, then we would expect the positive selection imposed by each interactor to result in strong positive selection on resistance $\left(H_{0}=\right.$ $\left.0.75=\beta_{1}+\beta_{2}-\beta_{0}\right)$. Rather, selection in the presence of both species was weak $\left(\beta_{12}=-0.04 \pm 0.05\right)$. Strong selection imposed by insects and Medicago was offset by strong nonadditive selection in the opposite direction $\left(\beta_{\text {nonadditive }}=\beta_{12}-\beta_{1}-\beta_{2}+\beta_{0}=-0.80 \pm 0.39 ;\right.$ Eq. 5), indicating that the effects of insects and Medicago were not independent. Plants experiencing both competition and herbivory had very low fitness and low variation in fitness (Table 2), potentially leading to little selection due to a lack of variation in fitness. In sum, indirect ecological effects cause selection on Acmispon resistance to be weaker in the presence of both Medicago and insect herbivores than would be expected in the absence of indirect ecological effects.

In contrast to selection on resistance, selection on tolerance was largely additive (nonsignificant tolerance Medicago $\times$ insecticide interaction; Table 1, Fig. 2B). There was selection against tolerance in the absence of insects and Medicago $\left(\beta_{0}=-0.57 \pm 0.33\right)$, suggesting a cost of tolerance. Selection was similar when Medicago 
TABLE 2. Mean ( \pm SE) relative fitness (seed number) based on family means in response to the effects of Medicago, insects, both Medicago and insects, or neither interactor.

\begin{tabular}{lc}
\hline \hline \multicolumn{1}{c}{ Treatment } & Relative fitness \\
\hline No Medicago or insects & $1.47 \pm 0.26$ \\
Insects only & $1.28 \pm 0.21$ \\
Medicago only & $1.07 \pm 0.19$ \\
Medicago plus insects & $0.18 \pm 0.05$ \\
\hline
\end{tabular}

was present $\left(\beta_{2}=-0.64 \pm 0.22\right)$, but weaker and positive when insects were present $\left(\beta_{1}=0.10 \pm 0.24\right)$. Equations 1 and 2 reveal that insects imposed strong selection for increased tolerance $\left(\beta_{\text {insects }}=\beta_{1}-\beta_{0}=0.67 \pm 0.33\right)$, but Medicago was not a significant selective agent on tolerance $\left(\beta_{\text {Medicago }}=\beta_{2}-\beta_{0}=-0.06 \pm 0.33\right.$; Fig. $\left.2 \mathrm{~B}\right)$. When both Medicago and insects were present, selection on tolerance tended to be slightly stronger than when only insects were present $\left(\beta_{12}=0.25 \pm 0.06 ; H_{0}=0.03\right)$. However, the strength of nonadditive selection was weak relative to the pairwise effect of insects and not significantly different from zero $\left(\beta_{\text {nonadditive }}=0.21 \pm\right.$ $0.46)$. This suggests that pairwise selection by insects on tolerance is most important for the evolution of tolerance in this population. The presence of Medicago did not significantly alter selection on tolerance imposed by insects.

\section{Case study 2: nonadditive selection in response to multiple pollinators}

Sahli and Conner (2011) investigated selection on floral traits in Raphanus raphanistrum by three species of pollinators (honey bees, bumble bees, and cabbage butterflies) individually and all together (combined treatment). There was no treatment with saturating hand pollination as discussed in Experimental design: Manipulating multiple species, but the selection measured is likely to be caused by the pollinators (i.e., $\beta_{0} \approx$ 0 ) for several reasons. First, selection was estimated on two anther position traits: anther exsertion, defined as the distance the long stamen anthers protrude out of the corolla tube, and anther dimorphism, the difference in the lengths of the short and long stamens within each flower. Second, selection was through male fitness, estimated as seed siring success through molecular genetic paternity analysis; selection on anther position traits through differences in seed siring success is most likely to be caused by pollinators. Last, measurements were taken on potted plants maintained in a greenhouse, with pollination occurring in large flight cages, so the plants were not exposed to most of the other selective agents they would normally encounter in the field environment.

Because this was not a fully factorial experiment, a full ANCOVA approach cannot be applied. Instead, we used planned contrasts in an ANCOVA framework to test for nonadditive selection. We constructed a contrast that compared the additive null hypothesis (the sum of the selection gradients in the three individual pollinator treatments) to the selection gradient in the combined treatment $\left(H_{0}: \beta_{\text {all }} 3\right.$ pollinators $=\beta_{\text {honeybees }}+\beta_{\text {bumblebees }}+$ $\beta_{\text {butterflies }}-2 \beta_{0}$; SAS code in the Supplement). Note that here we assume $\beta_{0}=0$. This approach is similar to that taken by Sahli and Conner (2011), but they used the mean of the selection gradients from the three individual treatments, rather than the sum, as a null hypothesis to test against the combined treatment. Also, Sahli and Conner used values of selection gradients that were weighted by visitation rates in individual treatments to correct for differences in visitation rates in the experimental flight cages. We use an unweighted sum because a key mechanism of nonadditive selection may be interactions among pollinators that change relative visitation rates. The choice of whether or not to weight values when summing selection gradients will depend on the specific experimental design and question being asked. For most cases, however, we do not recommend using weighted sums because the indirect ecological effects underlying nonadditive selection may be densitymediated. Accordingly, weighting sums based on the abundance of interactors would negate one of the key ecological mechanisms likely to cause the indirect ecological effects driving nonadditive selection.

The significant planned contrast comparing selection on exsertion in the presence of all species to the additive null hypothesis indicates nonadditive selection on exsertion $\left(F_{19,218}=8.97, P=0.003\right)$. The selection gradients in the individual treatments (Table 3) show that there was positive directional selection on anther exsertion in each of the individual treatments (not significant for butterflies), but there was essentially no selection in the combined treatment. Therefore, an unknown interaction among the pollinators when they were all present together rendered anther exsertion neutral in determining male seed siring success, as the nonadditive selection gradient $\left(\beta_{\text {nonadditive }}=-0.35 ; \mathrm{Eq}\right.$. 5 , assuming $\beta_{0}=0$ ) was stronger and opposed to each of the pairwise selection gradients. This cannot be attributed to a lack of variance in male fitness in the combined

TABLE 3. Results of the reanalysis of Sahli and Conner (2011) showing selection gradients on anther exsertion and dimorphism in wild radish in response to each of three pollinators, and the combination of all three pollinators.

\begin{tabular}{lrrrr}
\hline \hline $\begin{array}{l}\text { Trait and pollinator } \\
\quad \text { treatment }\end{array}$ & $\beta$ & SE & $t$ & $P$ \\
\hline Exsertion & & & & \\
$\quad$ Honey bee & 0.12 & 0.06 & 2.03 & $\mathbf{0 . 0 4}$ \\
Bumble bee & 0.13 & 0.06 & 2.23 & $\mathbf{0 . 0 3}$ \\
Cabbage butterfly & 0.09 & 0.06 & 1.48 & 0.14 \\
All three pollinators & -0.01 & 0.06 & -0.18 & 0.85 \\
Dimorphism & & & & \\
Honey bee & -0.13 & 0.06 & -2.20 & $\mathbf{0 . 0 3}$ \\
Bumble bee & -0.03 & 0.06 & -0.54 & 0.59 \\
Cabbage butterfly & 0.01 & 0.06 & 0.16 & 0.88 \\
All three pollinators & 0.02 & 0.06 & 0.28 & 0.78 \\
\hline
\end{tabular}

Note: Boldface indicates significance of $P<0.05$. 
treatment, as the variance in fitness was very similar across treatment groups, and the combined treatment variance was slightly higher than the mean of the three individual pollinator treatments (Sahli and Conner 2011). Interactions among the pollinators weakened the selection imposed by each, which in this case would result in little or no adaptation of exsertion to any individual pollinator. Nonadditive selection makes it impossible to predict evolutionary dynamics of exsertion without considering the effects of all three species simultaneously.

In contrast, selection on dimorphism was additive. Although honey bees imposed significant selection to reduce dimorphism, selection in the presence of all pollinators was not significantly different from the predicted additive effect of all pollinators $\left(F_{20,218}=\right.$ $\left.2.10, P=0.15 ; \beta_{\text {nonadditive }}=0.17\right)$. Honey bees were the only significant selective agent and these statistical analyses suggest that selection on dimorphism by honey bees was not significantly altered by the presence of other pollinators; pairwise selection by honey bees is sufficient to predict selection in a multipollinator environment. This suggests that decreased dimorphism was adapted to honey bees regardless of the presence of the other two pollinators. However, it is important to consider that, as in the exsertion results, there was essentially no selection on dimorphism with all three pollinators present; the lack of significant nonadditive selection may be due to relatively low statistical power, as the $P$ value was marginal $(0.15)$.

\section{Conclusions}

Since Darwin's (1859) description of the tangled bank of species interactions, both ecologists and evolutionary biologists have appreciated that complex interactions in communities can have implications for understanding evolution in natural communities. Although ecologists have long appreciated the importance of indirect ecological effects, few have considered such indirect effects as an important mechanism affecting patterns of natural selection in multispecies communities (Walsh 2013). Understanding evolution in a multispecies context is an important step in connecting selection on traits in pairwise species interactions to trait evolution in natural communities composed of many interacting species. A significant selection gradient in the presence of multiple species (e.g., $\beta_{12}$ ) indicates that a trait is adaptive with respect to the particular combination of species comprising that community. Nonadditive selection is critical for understanding trait adaptation in diverse natural communities because indirect ecological effects can affect the extent to which traits are adapted to any single species in a community. Although a trait may not be well adapted to any particular species interaction, nonadditive selection on a trait indicates that the trait is adapted to the indirect ecological effects in the community.
Previously, evolutionary biologists sought to understand evolution in a community context by testing for diffuse evolution. Diffuse evolution can occur because of genetic correlations between traits that affect species interactions, or because of diffuse selection, in which selection on a trait when one species is present is different than when multiple species are present (Hougen-Eitzman and Rausher 1994, Iwao and Rausher 1997). Clearly nonadditive selection, in which one species alters the selection imposed by another, may be related to diffuse selection, in which selection in multispecies scenarios differs from selection in pairwise scenarios (Appendix C: Fig. C1). We agree with Haloin and Strauss (2008) that previous work has not adequately distinguished between diffuse and nonadditive selection. Nonadditive selection, driven by indirect ecological effects, is one mechanism that can lead to diffuse selection (Inouye and Stinchcombe 2001), although nonadditive selection does not necessarily result in diffuse selection (Haloin and Strauss 2008; Appendix C: Fig. C1C). Likewise, diffuse selection can occur even when selection is additive, a phenomenon that Strauss and Irwin (2004) termed "ecological pleiotropy" (Appendix C: Fig. C1B).

Community ecologists frequently measure mean fitness in factorial manipulations of multiple species, but few also report individual traits and fitness that would also allow for tests of nonadditive selection. Although few studies have tested for nonadditive selection, the two studies re-analyzed here and two previous studies that did not explicitly address nonadditive selection (Juenger and Bergelson 1998, Lankau and Strauss 2008) support the view that nonadditive selection may be common. Further, the strength of nonadditive selection in these studies was often sufficient to offset selection in the opposite direction imposed by direct species interactions. If such patterns are common, then evolutionary outcomes in multispecies communities are likely to be strongly influenced by indirect ecological effects, to be difficult to predict, and will vary across communities that differ in species composition.

\section{ACKNOWLEDGMENTS}

We are grateful to J. Stinchcombe, S. Strauss, and several anonymous reviewers for providing valuable insight into our views on diffuse and nonadditive selection in previous versions of the manuscript. Funding was provided by the National Science Foundation under grants awarded to C. P. terHorst (DMS-1312490), J. A. Lau (DEB-0918963), and J. K. Conner (DEB-0919452). This is contribution number 1595 from the Kellogg Biological Station.

\section{Literature Cited}

Abrams, P. A. 1992. Predators that benefit prey and prey that harm predators: unusual effects of interacting foraging adaptation. American Naturalist 140:573-600.

Ackerly, D. D., and W. K. Cornwell. 2007. A trait-based approach to community assembly: partitioning of species trait values into within- and among-community components. Ecology Letters 10:135-145. 
Caruso, C. M., S. L. Scott, J. C. Wray, and C. A. Walsh. 2010. Pollinators, herbivores, and the maintenance of flower color variation: a case study with Lobelia siphilitica. International Journal of Plant Sciences 171:1020-1028.

Conner, J. K., and D. L. Hartl. 2004. A primer of ecological genetics. Sinauer, Sunderland, Massachusetts, USA.

Darwin, C. 1859 . On the origin of species by means of natural selection. John Murray, London, UK.

Galen, C. 1996. Rates of floral evolution: adaptation to bumblebee pollination in an Alpine wildflower, Polemonium viscosum. Evolution 50:120-125.

Haloin, J. R., and S. Y. Strauss. 2008. Interplay between ecological communities and evolution: review of feedbacks from microevolutionary to macroevolutionary scales. Annals of the New York Academy of Sciences 1133:87-125.

Hereford, J., T. F. Hansen, and D. Houle. 2004. Comparing strengths of directional selection: how strong is strong? Evolution 58:2133-2143.

Hougen-Eitzman, D., and M. D. Rausher. 1994. Interactions between herbivorous insects and plant-insect coevolution. American Naturalist 143:677-697.

Inouye, B., and J. R. Stinchcombe. 2001. Relationships between ecological interaction modifications and diffuse coevolution: similarities, differences, and causal links. Oikos 95:353-360.

Iwao, K., and M. D. Rausher. 1997. Evolution of plant resistance to multiple herbivores: quantifying diffuse coevolution. American Naturalist 149:316-335.

Juenger, T., and J. Bergelson. 1998. Pairwise versus diffuse natural selection and the multiple herbivores of scarlet gilia, Ipomopsis aggregata. Evolution 52:1583-1592.

Kingsolver, J. G., H. E. Hoekstra, J. M. Hoekstra, D. Berrigan, S. N. Vignieri, C. E. Hill, A. Hoang, P. Gibert, and P. Beerli. 2001. The strength of phenotypic selection in natural populations. American Naturalist 157:245-261.

Lande, R., and S. J. Arnold. 1983. The measurement of selection on correlated characters. Evolution 37:1210-1226.

Lankau, R. A., and S. Y. Strauss. 2008. Community complexity drives patterns of natural selection on a chemical defense of Brassica nigra. American Naturalist 171:150-161.

Lau, J. A. 2008. Beyond the ecological: biological invasions alter natural selection on a native plant species. Ecology 89: $1023-1031$.

Lawler, S. P. 1993. Direct and indirect effects in microcosm communities of protists. Oecologia 93:184-190.

Litchman, E., and C. A. Klausmeier. 2008. Trait-based community ecology of phytoplankton. Annual Review of Ecology, Evolution, and Systematics 39:615-639.

MacColl, A. D. C.. 2011. The ecological causes of evolution. Trends in Ecology and Evolution 26:514-522.

Menge, B. A. 1995. Indirect effects in marine rocky intertidal interaction webs: patterns and importance. Ecological Monographs 65:21-74.

Miller, T. E. 1994. Direct and indirect species interactions in an early old-field plant community. American Naturalist 143: 1007-1025.

Mitchell-Olds, T., and R. G. Shaw. 1987. Regression analysis of natural selection: statistical inference and biological interpretation. Evolution 41:1149-1161.

Morrissey, M. B., and K. Sakrejda. 2013. Unification of regression-based methods for the analysis of natural selection. Evolution 67:2094-2100.
Petraitis, P. S. 1998. How can we compare the importance of ecological processes if we never ask, "Compared to what?". Pages 182-201 in W. J. Resetarits, Jr., and J. Bernardo, editors. Experimental ecology - issues and perspectives. Oxford University Press, Oxford, UK.

Rausher, M. D. 1992. The measurement of selection on quantitative traits: biases due to environmental covariances between traits and fitness. Evolution 46:616-626.

Sahli, H. F., and J. K. Conner. 2011. Testing for conflicting and nonadditive selection: floral adapation to multiple pollinators through male and female fitness. Evolution 65: $1457-1473$.

Stanton, M. L., and D. A. Thiede. 2005. Statistical convenience vs biological insight: consequences of data transformation for the analysis of fitness variation in heterogenous environments. New Phytologist 166:319-337.

Stinchcombe, J. R., and M. D. Rausher. 2001. Diffuse selection on resistance to deer herbivory in the ivyleaf morning glory, Ipomoea hederacea. American Naturalist 158:376-388.

Stinchcombe, J. R., and M. D. Rausher. 2002. The evolution of tolerance to deer herbivory: modifications caused by the density of insect herbivores. Proceedings of the Royal Society of London B 269:1241-1246.

Stone, L., and A. Roberts. 1991. Conditions for a species to gain advantage from the presence of competitors. Ecology 72:1964-1972.

Strauss, S. Y. 1991. Indirect effects in community ecology: their definition, study and importance. Trends in Ecology and Evolution 6:206-210.

Strauss, S. Y., and R. E. Irwin. 2004. Ecological and evolutionary consequences of multispecies plant-animal interactions. Annual Review of Ecology, Evolution, and Systematics 35:435-466.

Strauss, S. Y., H. Sahli, and J. K. Conner. 2005. Toward a more trait-centered approach to diffuse (co)evolution. New Phtyologist 165:81-90.

terHorst, C. P. 2010. Evolution in response to direct and indirect ecological effects in pitcher plant inquiline communities. American Naturalist 176:675-685.

terHorst, C. P. 2011. Experimental evolution of protozoan traits in response to interspecific competition. Journal of Evolutionary Biology 24:36-46.

terHorst, C. P., T. E. Miller, and D. R. Levitan. 2010. Discrepancies in evolutionary rates change ecological effect size of predators on prey. Ecology 91:629-636.

Vandermeer, J. H. 1969. Competitive structure of communities: an experimental approach with protozoa. Ecology 50:362371.

Violle, C., B. J. Enquist, B. J. McGill, L. Jiang, C. H. Albert, C. Hulshof, V. Jung, and J. Messier. 2012. The return of the variance: intraspecific variability in community ecology. Trends in Ecology and Evolution 27:244-252.

Wade, M. J., and S. Kalisz. 1990. The causes of natural selection. Evolution 44:1947-1955.

Walsh, M. R. 2013. The evolutionary consequences of indirect effects. Trends in Ecology and Evolution 28:23-29.

Wootton, J. T. 1994. The nature and consequences of indirect effects in ecological communities. Annual Review of Ecology, Evolution, and Systematics 25:443-466.

\section{Supplemental Material}

\section{Ecological Archives}

Appendices A-C and the Supplement are available online: http://dx.doi.org/10.1890/14-0619.1.sm 